\title{
DAS MEHRSPRACHIGE ABGEORDNETENHAUS DER TSCHECHOSLOWAKEI IN SEINER PARLAMENTARISCHEN PRAXIS 1920-1938
}

\author{
BENJAMIN CONRAD
}

JOHANNES GUTENBERG-UNIVERSITÄT MAINZ

\begin{abstract}
The Multilingual Chamber of Deputies of Czechoslovakia in its Parliamentary Practice, 1920-1938
\end{abstract}

In addition to the two official languages of Czech and Slovak, German, Hungarian, Russian, Ukrainian, and Polish were allowed to be spoken as secondary languages in the lower house of Czechoslovakia's parliament in 1920 in order to increase the acceptance of the new republic by the deputies of all national minorities. By swearing the oath in one of these languages, a deputy obtained the right to use this language in parliament. This article examines in which situations and how often members of parliament of minority descent took advantage of this right, regardless of their political convictions. The paper finds that although minority deputies, especially Hungarian or Polish ones, intensively took advantage of their right to participate in the debates in their languages, the use of German among German-speaking deputies progressively declined over the 1930s. This corresponded to the growing self-disintegration of Germans, especially after 1935.

Keywords: Czechoslovakia; Czechoslovak-German relations; 1918-1938; national minorities; language policy; parliament

DOI: $10.14712 / 23363231.2017 .2$

Dr. Benjamin Conrad ist wissenschaftlicher Mitarbeiter am Arbeitsbereich Osteuropäische Geschichte an der Johannes Gutenberg-Universität Mainz. Korrespondenzanschrift: Arbeitsbereich Osteuropäische Geschichte, Johannes Gutenberg-Universität, Philosophicum - Welderweg 18, 55099 Mainz.E-Mail-Adresse: conradb@uni-mainz.de. 


\section{Einleitung}

„Dazu der Sprachwirrwar [sic]: Es wurde in sechs verschiedenen Sprachen gesprochen, nämlich tschechisch, deutsch, slowakisch, ungarisch, ruthenisch und polnisch, die immer wieder nur von einem Teil der Mitglieder des Hauses verstanden wurden, die Staatssprache mit inbegriffen, die in ihre zwei Teile tschechisch und slowakisch zerfiel [...]", 1 erinnerte sich Felix von Luschka in den 1960er Jahren an das mehrsprachige Abgeordnetenhaus der Tschechoslowakischen Republik (ČSR). Luschka war 1920-1938 Abgeordneter der Deutschen Christlich-Sozialen Volkspartei (DCSVP) und dann 1938 kurzzeitig der Sudetendeutschen Partei (SdP) gewesen. Hierbei macht Luschka auf ein Problem des tschechoslowakischen Parlamentarismus der Zwischenkriegszeit aufmerksam: Um alle Wortmeldungen verstehen zu können, hätte ein Abgeordneter alle Sprachen verstehen müssen, was in der Praxis nicht der Fall war.

War das mehrsprachige Parlament in der Praxis tatsächlich derartig hinderlich? In manchen Publikationen wird die negative Darstellung Luschkas übernommen. Sie bildet einen Baustein in der Erklärung des Scheiterns der Tschechoslowakei der Zwischenkriegszeit insgesamt und ihres Parlamentarismus im Speziellen. ${ }^{2}$ Diese Einschätzung soll in der nachfolgenden Untersuchung hinterfragt werden. Zwar erscheint ein einsprachiges Parlament - aufgrund der von Luschka angesprochenen Teilung der Staatssprache Tschechoslowakisch in die beiden Teile Tschechisch und Slowakisch wäre es im Falle der ČSR in der Praxis ein zweisprachiges Parlament geworden - praktikabler. Doch dem stünde auch ein gewichtiges Gegenargument gegenüber: Aufgrund der mangelnden Sprachkenntnisse der großen Mehrheit der Abgeordneten der neuen Minderheiten wären diese zumindest in den Anfangsjahren mit noch größeren Hürden bei der Beteiligung konfrontiert gewesen. Ausgehend von der negativen Einschätzung Luschkas möchte dieser Aufsatz daher einen Beitrag zur Kulturgeschichte des Parlamentarismus leisten, indem er das Abgeordnetenhaus - das Unterhaus des Parlaments der ČSR der Zwischenkriegszeit - unter dem Aspekt des Sprachgebrauchs analysiert.

1 Felix von Luschka, „Im Parlament der Ersten Tschechoslowakischen Republik. Erinnerungen eines sudetendeutschen Abgeordneten 1920-1938“, Bohemia 4 (1963): 228-74, hier 234-35.

2 So Werner Dietl, Die Deutsche Christlichsoziale Volkspartei in der Ersten Tschechoslowakischen Republik (1918-1929) (München: Sudetendeutsches Archiv, 1991), 241; Jaroslav Kučera, Minderheit im Nationalstaat. Die Sprachenfrage in den tschechisch-deutschen Beziehungen 1918-1938 (München: Oldenbourg, 1999), 213. 
International folgten wenige Parlamente der Idee der Mehrsprachigkeit: Gemeinsam mit Finnland und Lettland war die ČSR einer von nur drei neuen Staaten im Osten Europas, in denen die Benutzung von mehr als einer Sprache im Parlament dauerhaft gestattet war. Allerdings waren in Finnland mit Finnisch und Schwedisch sowie in Lettland mit Lettisch, Deutsch und Russisch nur zwei und drei Sprachen dauerhaft zugelassen - und nicht sieben ${ }^{3}$ wie in der ČSR. Der Parlamentarismus Lettlands 1918-1934 wird in diesem Aufsatz als Referenz herangezogen. Zwar sind beide Parlamente von der Größe her sehr unterschiedlich gewesen, ansonsten gab es neben der Mehrsprachigkeit aber noch zahlreiche andere Gemeinsamkeiten. Beide Parlamente eint, dass sie die obersten legislativen Organe von 1918 neugegründeten Staaten waren. In beiden Staaten hatten Angehörige der späteren nationalen Minderheiten vorher die bestimmenden Nationalitäten dargestellt. Ihre Vertreter waren an den neuen Status als Minderheit also nicht gewöhnt. Besonders wichtig aber ist, dass die Bestimmungen, in denen die Mehrsprachigkeit im Parlament zum Ausdruck kommen konnte, ausgesprochen ähnlich waren. Deshalb wird hin und wieder auf Lettland zurückgekommen, um die Spezifika der Entwicklung in der ČSR genauer zu konturieren. ${ }^{4}$

Der Beitrag klärt zuerst in aller Kürze den rechtlichen Rahmen der Mehrsprachigkeit des Abgeordnetenhauses der ČSR. Dabei werden die Abgeordneten nach der Ableistung des Eides in die Sprachgruppen eingeteilt. Dieses formale Kriterium rührt aus der Geschäftsordnung von 1920. Anschließend werden Entwicklungen und Tendenzen innerhalb der Sprachgruppen benannt, um sich abschließend der Grundfrage zu widmen, ob die Mehrsprachigkeit des Parlaments, immerhin des formell wichtigsten legislativen Organs im Staat, tatsächlich derartig problematisch war oder ob es einen Weg zur besseren Teilnahme der politischen Vertreter der Minderheiten darstellen konnte.

Die Wichtigkeit dieser Frage wurde bereits von Jaroslav Kučera erkannt, der in seinem 1999 erschienenen Werk die Frage, ob der Lösungsansatz im Abgeordnetenhaus der ČSR gelungen war, verneint. Zwar beurteilt Kučera den mehrsprachigen Ansatz an sich positiv, der in der ČSR gefundene Modus sei jedoch für die Minderheiten zu einengend gewesen. ${ }^{5}$ Eindeutiger Schwerpunkt

3 Luschka fasst in seinem eingangs erwähnten Zitat Ukrainisch und Russisch zu „Ruthenisch“ zusammen. In Luschkas Form übernommen von Kučera, Minderheit, 213.

4 Die Informationen zu Lettland entstammen dem Werk Benjamin Conrad, Loyalitäten, Identitäten und Interessen. Deutsche Parlamentarier im Lettland und Polen der Zwischenkriegszeit (Göttingen: Vandenhoeck \& Ruprecht, 2016), passim.

5 Kučera, Minderheit, 210, 213. 
der Untersuchung Kučeras ist der rechtliche Rahmen. Da in der vorliegenden Studie die Praxis untersucht wird, wird sie sich nicht mit Kučeras Einschätzungen auseinandersetzen.

Datengrundlage dieses Aufsatzes ist eine eigens vorgenommene Durchsicht der elektronischen Version des Registers des Abgeordnetenhauses 1920-1938. ${ }^{6}$ Die Durchsicht und Zählung jeder Sitzung des Abgeordnetenhauses war ein zeitraubendes Unterfangen, schließt andererseits aber Fehlschlüsse aufgrund unvollständiger Daten aus. Der gewonnene Datensatz ermöglicht auch zahlreiche deskriptiv-statistische Erkenntnisse. Eine vollständige Durchsicht aller Stenogramme und ebenso der Sitzungen der Ausschüsse stellt ein Desiderat der Forschung dar, aus dem im Detail weitere Erkenntnisse gewonnen werden könnten.

\section{Zum Sprachenrecht im Abgeordnetenhaus der Tschechoslowakei}

Wie Lettland verfügte auch die ČSR 1918-1920 über ein ernanntes Parlament, die Tschechoslowakische Nationalversammlung (Národní shromáždění československé). Sie bestand ausschließlich aus Tschechen und Slowaken. Anders als die lettischen Parteien waren ihre tschechoslowakischen Pendants zunächst nicht bereit, den anderen Nationalitäten die Teilnahme einzuräumen, obwohl Regierung und Nationalversammlung der ČSR weitreichende territoriale Ansprüche erhoben hatten. Deshalb waren 1918-1920 zunächst nur Tschechisch und Slowakisch Parlamentssprachen. In der damaligen Terminologie, die dem Gedanken des Tschechoslowakismus folgte, handelte es sich bei Tschechisch und Slowakisch um "Zweige“ einer postulierten gemeinsamen tschechoslowakischen Sprache. ${ }^{7}$ In der parlamentarischen Praxis waren es aber zwei verschiedene Sprachen, wie im eingangs erwähnten Zitat Luschkas zutreffend genannt.

Bei der Ausgestaltung des Parlamentarismus in der ČSR, die die Nationalversammlung trotz ihrer mangelnden demokratischen Legitimation vornahm, wurde durch die Geschäftsordnung vom 15. April 1920 das Prinzip der Mehrsprachigkeit erneuert, obwohl vor 1914 gerade mehrsprachige Parlamente, wie

6 Vgl. „Společná česko-slovenská digitální parlamentní knihovna“, offizielle Seite des Abgeordnetenhauses des Parlaments der Tschechischen Republik, letzter Zugriff 02.05.2016, www.psp.cz/sqw /hp.sqw?k=82. In diesem Beitrag erfolgen Anmerkungen im Bereich der Stenogramme nur bei Bezugnahme auf bestimmte Sitzungen.

7 Janko Prunk und Marjetka Rangus, „Macht und Ohnmacht des Parlamentarismus bei der Lösung der nationalen Frage in Vielvölkerstaaten im 19. und 20. Jahrhundert“, in Parlamentarische Kulturen in Europa. Das Parlament als Kommunikationsraum, hrsg. v. Andreas Schulz und Andreas Wirsching (Düsseldorf: Droste, 2012), 285-300, hier 291. 
der österreichische Reichsrat und der böhmische Landtag, dysfunktional gewesen waren. Für diese Dysfunktionalität war allerdings nicht die Mehrsprachigkeit an sich verantwortlich gewesen, sondern nicht zuletzt große Teile der tschechischen Abgeordnetengruppe durch beständige Obstruktion. Die Geschäftsordnung des Abgeordnetenhauses von 1920 erschwerte die Obstruktion erheblich und machte sie in der Praxis fast unmöglich. ${ }^{8}$

Wie im benachbarten Polen waren ab 1920 alle gewählten Abgeordneten verpflichtet, einen Amtseid zu leisten. Besagter Eid war bereits vor Zusammentritt des ersten gewählten Abgeordnetenhauses (Poslanecká sněmovna) in der Geschäftsordnung festgelegt worden. $\$ 49$ (6) gestand den Abgeordneten „deutscher, russischer (kleinrussischer), ungarischer oder polnischer Nationalität“" die Ableistung des Eides in der Sprache ihrer Nationalität zu. Ebendiese Nationalität hatten sie vorher gemäß $₫ 6$ bei der Kanzlei des Abgeordnetenhauses zu hinterlegen, was 1925 noch einmal präzisiert wurde, nachdem es zu Doppelnennungen von Nationalitäten gekommen war. Selbstverständlich stand den Abgeordneten auch die Leistung des Eides in der Staatssprache offen. Allerdings beinhaltete die Abgabe des Eides auf Tschechisch oder Slowakisch die Verpflichtung, sich im Plenum fortan ausschließlich in dieser Sprache zu äußern. Abgeordnete, die in einer Minderheitensprache den Eid leisteten, konnten dagegen zwischen dieser und der Staatssprache wechseln. ${ }^{10}$

Diese Praxis führte dazu, dass für fast alle Parlamentarier der Minderheiten kein Hindernis bestand, ihren Eid in der Minderheitensprache abzugeben. Für die Abgeordneten der deutschen Bevölkerungsgruppe galt dies sogar ausnahmslos. Die symbolische Ableistung des Eides in der Muttersprache in aller Öffentlichkeit wurde zu einem unverzichtbaren symbolischen Beweis der Zugehörigkeit zur entsprechenden Nationalität.

Tschechoslowakisch und die Minderheitensprachen waren nicht gleichberechtigt: Reden auf Deutsch, Polnisch, Russisch, Ukrainisch und Ungarisch wurden im Protokoll meist auf Tschechisch abgedruckt, mit Ausnahme der auf

8 Sbirka zákonů a nařizení státu československého, Nr. 325 (1920): 753-54; Luschka, „Parlament“, 232.

9 Sbirka zákonů a nařízeni státu československého, Nr. 325 (1920): 746, 753.

10 Werner Glück, Sprachenrecht und Sprachenpraxis in der Tschechoslowakischen Republik nach dem Stande von 1938 (Halle [Saale]: Niemeyer, 1939), 175; Kučera, Minderheit, 212; Norbert Linz, Der Bund der Landwirte in der Ersten Tschechoslowakischen Republik (München: Oldenbourg, 1972), 260. Bei Zwischenrufen gebrauchten Abgeordnete der Staatssprachen aber sehr wohl Minderheitensprachen, so der Linkssozialist Heřman Tausik am 11. März 1921, vgl. I. Abgeordnetenhaus: 63. Sitzung, 11. März 1921. 
Ungarisch gehaltenen Reden, die sich generell auf Slowakisch finden. ${ }^{11} \mathrm{Nach}$ dem Rednernamen steht im Protokoll ein Vermerk der Originalsprache (němec$k y$, polsky, rusky, malorusky, mad'arsky). Entsprechend der Terminologie des Zarenreichs wurde Ukrainisch konservativ weiter „Kleinrussisch“ (malorusky) genannt, wie auch beim Druck der Eidesformeln im Russischen unter Ignoranz der sowjetischen Rechtschreibreform konsequent an der zarischen Rechtschreibung festgehalten wurde. Im Plenum war die freie Wahl der Sprache nur bei den Wortmeldungsformen „Rede in der Debatte“ ( (prohlášenî) und „Anmerkungen in der Sache“ (věcná poznámka) möglich. Alle anderen Wortbeiträge sollten ausschließlich in der Staatssprache erfolgen.

Vorstöße der deutschen Abgeordneten, die Geschäftsordnung einer Revision zu unterziehen, scheiterten erstmalig im Jahr 1921. Aufgrund der ablehnenden Haltung vieler deutscher Abgeordneter gegenüber der ČSR erteilte selbst der konzessionsbereite Teil der tschechisch- und slowakischsprachigen Parlamentarier einer Revision eine Absage. Ein zweiter Vorstoß wurde im Zusammenhang mit dem Beginn des deutschen Aktivismus 1926-1927 unternommen, erbrachte aber ebenso keine Resultate. In beiden Fällen zielten die Initiativen nicht darauf ab, das Deutsche als Staatssprache anzuerkennen, wohl aber in der parlamentarischen Praxis konkrete Verbesserungen herbeizuführen. Ein Antrag der SdP, der DCSVP und ungarischer Politiker zeitigte 1935 genauswenig Erfolge wie entsprechende Vorschläge der deutschen aktivistischen Parteien von 1937. ${ }^{12}$ Zuletzt forderten Wenzel Jaksch und Siegfried Taub von der Deutschen Sozialistischen Arbeiterpartei (DSAP) - der letzten noch verbliebenen aktivistischen Partei - im August 1938 gegenüber dem britischen Sondergesandten Walter Runciman die volle parlamentarische Gleichberechtigung der deutschen Sprache. ${ }^{13}$

\section{Individuelle Eidleistung als Sprachzugehörigkeitsangabe 1920-1938}

Divergierende Angaben zur Größe der Abgeordnetengruppen stellen auch für die ČSR ein vielfach auftretendes Problem in Quellen und Literatur dar. Zeitgenössisch und auch nach dem Zweiten Weltkrieg wurde eine Unterteilung

11 Wird Glück gefolgt, so seien sogar ausnahmslos alle Reden ins Tschechische übersetzt worden. Für auf Ungarisch gehaltene Wortmeldungen ist dies allerdings nicht zutreffend. Vgl. Glück, Sprachenrecht, 177. Korrekt: Kučera, Minderheit, 213.

12 Kučera, Minderheit, 216-20.

13 Eva Broklová, Politická kultura německých aktivistických stran v Československu 1918-1938 (Praha: Karolinum, 1999), 31-32. 
gerne nach der Parteizugehörigkeit vorgenommen. Weil aber ein multinationaler Staat auch multinationale Parteien nach sich zog, konnten somit Tschechen und Slowaken kaum voneinander getrennt werden. Verschlimmernd wurden multinationale Parteien der Minderheiten meist der Sprachgruppe mit dem größten Anteil zugeschlagen, was die Unschärfe weiter erhöht. Bei der Kommunistischen Partei der Tschechoslowakei (Komunistická strana Českosloven-

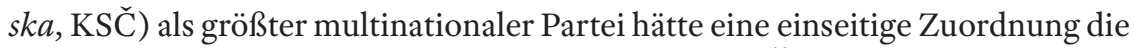
Statistik völlig unbrauchbar gemacht, weshalb die KSČ gerne unberücksichtigt blieb. 1925 gaben von 41 Kommunisten 21 den Eid auf Tschechisch, acht auf Deutsch, sechs auf Slowakisch, drei auf Ungarisch, zwei auf Ukrainisch und einer auf Polnisch ab. Somit war die KSČ-Fraktion zu Beginn der Legislaturperiode - es verließen bis 1929 zahlreiche Abgeordnete die Fraktion - in sprachlicher Hinsicht eine Tschechoslowakei im Kleinen. ${ }^{14}$

Gleich zu Beginn der I. Legislaturperiode beklagten die konservativen und agrarischen deutschen Abgeordneten der ČSR im Zusammenhang mit ihrer Denkschrift an den Völkerbund die unterdurchschnittliche Repräsentanz der Deutschen im Abgeordnetenhaus. Diese Behauptung konkretisierten sie anhand der Nationalitätenverteilung: 1920 habe es angeblich 199 Tschechoslowaken, 72 Deutschen und zehn Magyaren gegeben. Argumentativ verursacht dies übrigens schon an dieser Stelle das Problem, das selbst 72 deutsche Abgeordnete einem Anteil von 25,6 \% entsprochen hätten, während die auch in der Denkschrift zitierte Volkszählung von 1921 einen Anteil von 23,4 \% Staatsangehöriger deutscher Nationalität angab. ${ }^{15}$

Die aus der genannten, methodisch problematischen Zählweise generierte Zahl von 72 genoss in der Literatur eine bemerkenswerte Persistenz, ${ }^{16}$ dies zu

${ }^{14}$ II. Abgeordnetenhaus: 1. Sitzung, 17. Dezember 1925; 7. Sitzung, 26. Februar 1926; 29. Sitzung, 10. Juni 1926. Alfred Bohmann nennt irrtümlich elf deutsche Kommunisten, vgl. Alfred Bohmann, Menschen und Grenzen, Bd. 4, Bevölkerung und Nationalitäten in der Tschechoslowakei (Köln: Wissenschaft und Politik, 1975), 174. Korrekt: Helmut Slapnicka, „Die böhmischen Länder und die Slowakei 1919-1945“, in Handbuch der Geschichte der böhmischen Länder, hrsg. v. Karl Bosl, Bd. 4 (Stuttgart: Hiersemann, 1970), 3-150, hier 27. Slapnicka fasst allerdings Tschechen und Slowaken zusammen.

15 Deutsche Parlamentarier an den Völkerbund. Eine Ergänzung der im Sommer 1922 dem Völkerbund überreichten Denkschrift deutscher Parlamentarier (Prag: Verlag der Deutschpolitischen Arbeitsstelle, 1922), 38-42.

16 Zdeněk Kárník, České země v éře První republiky (1918-1938), Bd. 1 (Praha: Libri, 2000), 126; Jörg Kracik, Die Politik des deutschen Aktivismus in der Tschechoslowakei 1920-1938 (Frankfurt a. M.: Lang, 1999), 73; Kučera, Minderheit, 214; Linz, Bund der Landwirte, 214, 241; Slapnicka, „Länder“, 24; Gábor Szent-Ivány, Graf János Esterházy (Wien: Böhlau, 1995), 51; Mit Einschränkungen: Dietl, Deutsche Christlichsoziale Volkspartei, 200-2. 
Unrecht: Vier Abgeordnete deutsch-ungarischer Parteien, nämlich Johann Jabloniczky und Johann Tobler von der Christlich-Sozialen Landespartei (Országos Keresztényszocialista Párt, OKSZP) sowie Samuel Mayer und Paul Wittich von der Deutsch-Ungarischen Sozialdemokratischen Partei, die ihren Amtseid auf Deutsch abgaben und vor dem Unterhaus stets Deutsch sprachen, zählten dabei zu den Ungarn oder zu den Tschechoslowaken. Das Verdienst Mads Ole Ballings, in seinem Handbuch ist es, erstmalig auch alle gemischtnationalen Parteien der ČSR auf deutsche Abgeordnete hin untersucht zu haben, weshalb dieses Werk fehlerfrei die deutschsprachigen Abgeordneten in der ČSR auflistet. ${ }^{17}$

Es ist daher zielführend, die Legislaturperioden auf die Amtseide aller Nationalitäten hin zu untersuchen, zumal die Eide in der Tendenz eine individuelle Selbstzuschreibung darstellen, ihnen also nicht im Nachhinein eine solche zugeschrieben werden muss. Auf wenige Ausnahmen pragmatischer Natur im Bereich der ungarischen Abgeordneten wird noch eingegangen. Zu Beginn der ersten Legislaturperiode 1920 waren zunächst vier Sprachgruppen vertreten: Von 281 Abgeordneten gaben 156 den Eid auf Tschechisch (55,5 \%), 76 auf Deutsch (27\%), 41 auf Slowakisch (14,6 \%) und acht auf Ungarisch $(2,8 \%)$ ab. Durch die Nachwahl der heimgekehrten Tschechoslowakischen Legion erhöhte sich die Zahl der tschechischsprachigen Abgeordneten am 24. Juni 1921 um vier. Im April 1924 traten neun am 16. März 1924 im Wahlkreis XXII Karpatenland gewählte Abgeordnete hinzu, von denen drei den Eid auf Russisch, je zwei auf Ukrainisch und Ungarisch und je einer auf Tschechisch und Slowakisch ablegten. ${ }^{18}$ Einschließlich aller bis April 1924 geschehenen Sprachwechsel durch Mandatsänderungen hatte das Parlament nun 294 Mitglieder, von denen 162 den Eid auf Tschechisch (55,1 \%), 75 auf Deutsch (25,5 \%), 42 auf Slowakisch (14,3\%), zehn auf Ungarisch (3,4 \%), drei auf Russisch (1,0\%) und zwei auf Ukrainisch $(0,7 \%)$ abgelegt hatten. Damit waren ab 1924 auch die bereits 1920 in der Geschäftsordnung vorgesehenen Sprachen Russisch und Ukrainisch erstmalig auch tatsächlich vertreten.

Zu Beginn der II. Legislaturperiode zählte das Abgeordnetenhaus 300 Parlamentarier, von denen 160 den Eid auf Tschechisch (53,3\%), 75 auf Deutsch (25\%), 47 auf Slowakisch (15,7\%), zehn auf Ungarisch (3,3\%), fünf auf

${ }_{17}$ Mads Ole Balling, Von Reval bis Bukarest. Statistisch-biographisches Handbuch der Parlamentarier der deutschen Minderheiten in Ostmittel- und Südosteuropa 1919-1945, Bd. 1 (Kopenhagen: Dokumentation-Verlag, 1991), 252-84. Dietl nennt Tobler dagegen einen „ungarischen Christlichsozialen“. Vgl. Dietl, Deutsche Christlichsoziale Volkspartei, 225.

18 I. Abgeordnetenhaus: 69. Sitzung, 24. Juni 1921; 258. Sitzung, 8. April 1924; 261. Sitzung, 14. April 1924. 
Ukrainisch (1,7 \%), zwei auf Polnisch (0,7 \%) und einer auf Russisch $(0,3 \%)$ ablegten. ${ }^{19}$ Damit war mit dem Polnischen auch die letzte in der Geschäftsordnung vorgesehene Sprache erstmalig vertreten. Die II. Legislaturperiode 1925-1929 war die einzige, in denen Veränderungen nur durch individuelle Ereignisse - wie Mandatsverzichte oder Todesfälle - zustande kamen, weshalb es lediglich sehr geringe Verschiebungen gab.

Nach der vorgezogenen Neuwahl zur III. Legislaturperiode legten im Dezember 1929166 Parlamentarier den Eid auf Tschechisch (55,3 \%), 74 auf Deutsch (24,7\%), 44 auf Slowakisch (14,7\%), sieben auf Ungarisch $(2,3 \%)$ und je drei auf Polnisch, Russisch und Ukrainisch (je $1 \%$ ) ab. In dieser Legislaturperiode kam es zu einer signifikanteren Änderung, als am 28. November 1933 acht Mandate aufgrund des vorangegangenen Verbots der Deutschen Nationalsozialistischen Arbeiterpartei (DNSAP) annulliert wurden. ${ }^{20}$ Einschließlich aller bislang erfolgten Sprachwechsel hatte das Abgeordnetenhaus nun noch 292 Abgeordnete, davon waren 166 tschechisch- (56,8 \%), 66 deutsch- (22\%), 45 slowakisch- $(15,4 \%)$, sieben ungarisch- $(2,4 \%)$, je drei polnisch- und russischsprachig (je $1 \%$ ) sowie zwei ukrainischsprachig $(0,7 \%)$.

Von den ersten der 1935 gewählten Abgeordneten der IV. Legislaturperiode waren 161 tschechisch- (53,7\%), 72 deutsch- (24\%), 47 slowakisch- (15,7\%), elf ungarisch- $(3,7 \%)$, fünf russisch- $(1,7 \%)$ und je zwei polnisch- und ukrainischsprachig (je 0,7 \%). ${ }^{21} 1938$ ergaben sich einschneidende Veränderungen, nachdem in Folge des Münchener Abkommens, der Abtretung des Olsagebiets an Polen und des ersten Wiener Schiedsspruches insgesamt 72 Mandate entfielen. Dabei behielten Abgeordnete tschechischer, slowakischer und ruthenischer Nationalität ihr Mandat, auch wenn sie im Abtretungsgebiet gelebt hatten. Das im September 1938 durch die tschechoslowakische Regierung ausgesprochene

19 Diese Zahlen nannte korrekt Gotthold Rhode, „Die Tschechoslowakei von 1918 bis 1939“, Aus Politik und Zeitgeschichte 49 (1962): 637. Gustav Peters und Alois Erben nennen korrekt 74 Deutsche, aber unzutreffend neun Magyaren, einen Polen und einen Ruthenen. Siehe Gustav Peters und Alois Erben, Die Deutschen in der Tschechoslowakei (Berlin: Deutscher Schutzbund-Verlag, [1925]), 11. Manfred Alexander nennt unzutreffend vier Ungarn, einen Polen und allein ohne Kommunisten 71 (!) Deutsche, wobei diese zu hohe Ziffer auf einem Fehler bei der Zählung der BdL-Parlamentarier beruht. Vgl. Manfred Alexander, „Leistungen, Belastungen und Gefährdungen der Demokratie in der Ersten Tschechoslowakischen Republik“, Bohemia 27 (1986): 72-87, hier 75.

20 III. Abgeordnetenhaus: 300. Sitzung, 28. November 1933. Der DNSAP-Abgeordnete Hans Krebs hatte sein Mandat bereits am 3. Oktober 1933 niedergelegt, ohne dass es nachbesetzt worden wäre, vgl. III. Abgeordnetenhaus: 293. Sitzung, 17. Oktober 1933.

21 Slapnicka behauptet falsch, 1935 hätten „weder Polen noch Juden ein Mandat“ erzielt. Siehe Slapnicka, „Länder“, 26. 
SdP-Verbot zog übrigens unter dem Druck Hitlers anders als das DNSAP-Verbot 1933 keine Konsequenzen nach sich: Die verbliebenen Deputierten der ehemaligen SdP-Fraktion setzten ihre Tätigkeit als NSDAP-Fraktion fort. Als das Abgeordnetenhaus am 17. November 1938 erstmalig wieder zu einer Sitzung zusammenkam, hatte es somit noch 228 Mitglieder, davon 161 tschechisch(70,6\%), 46 slowakisch- (20,2\%), zwölf deutsch- (5,3\%), fünf russisch- (2,2\%), drei ukrainisch- $(1,3 \%)$ und einer ungarischsprachig $(0,4 \%)$. Polnischsprachige Abgeordnete gab es keine mehr. ${ }^{22}$

Durch das Verbot der KSČ wurden am 28. Dezember 1938 weitere 25 Mandate annulliert. Nunmehr hatte das Abgeordnetenhaus nur noch 203 Mitglieder, davon 145 tschechisch- (71,4\%), 41 slowakisch- (20,2\%), zehn deutsch- $(4,9 \%)$, fünf russisch- (2,5\%) und je eins ukrainisch- und ungarischsprachig (je $0,5 \%$ ). Dies spielte allerdings in der Praxis keine Rolle mehr, da nach der Sitzung am 16. Dezember $1938^{23}$ das Abgeordnetenhaus zu keiner Sitzung mehr zusammengerufen wurde.

\section{Entwicklungen und Tendenzen}

Durch die Analyse des Sprachgebrauchs im Abgeordnetenhaus der ČSR zeigt sich, dass in der Ersten Republik nur zwei der sieben Sprachgruppen stabil vertreten waren, nämlich das Slowakische mit 41-47 Parlamentarier (14,3$15,7 \%$ ) und das Polnische mit zwei bis drei Abgeordneten (0,7-1 \%). Im Grunde trifft das Kriterium der Stabilität aber auch auf das Tschechische mit 156-168 ${ }^{24}$ (53,3-57,5\%) und das Deutsche mit 66-76 Abgeordneten (22-27\%) zu, weil die Bandbreite der Schwankungen auf Eingriffe in das politische System zurückzuführen ist. Diese Eingriffe stärkten stets das tschechische Element, während die deutschsprachige Abgeordnetengruppe meist geschwächt wurde. Werden nur die Wahlen 1925, 1929 und 1935 ohne das DNSAP-Verbot berücksichtigt, so lag der Anteil der tschechischsprachigen Parlamentarier bei 53,3-56 \% und der der deutschsprachigen bei 24-25\% - und ist damit ebenfalls stabil zu nennen.

\footnotetext{
22 IV. Abgeordnetenhaus: 151. Sitzung, 17. November 1938; 157. Sitzung, 14. Dezember 1938. Jörg K. Hoensch, Geschichte der Tschechoslowakei (Stuttgart: Kohlhammer, 1992), 91, 100. Ladislav Lipscher, „Die magyarischen, polnischen, ruthenischen und jüdischen Parteien in der ČSR“, in Die Erste Tschechoslowakische Republik als multinationaler Parteienstaat, hrsg. v. Karl Bosl (München: Oldenbourg, 1979), 217-40, hier 227.

23 IV. Abgeordnetenhaus: 159. Sitzung, 16. Dezember 1938.

${ }^{24}$ 1934-1935 waren 168 von 292 Abgeordneten tschechischsprachig (57,5\%).
} 
Instabiler war dagegen mit sieben bis elf (2,3-3,7 \%) die Anzahl der ungarischsprachigen Parlamentarier. Diese Schwankung lässt sich nicht primär auf Eingriffe in das politische System zurückführen. Verantwortlich dafür war, dass die Politiker der ungarischen Bevölkerungsgruppe gerne in gemischtnationalen Parteien antraten. Dazu kam, dass viele auf der OKSZP-Liste gewählte Politiker den Amtseid nicht auf Ungarisch abgaben: 1920 gaben von fünf Abgeordneten drei den Eid auf Ungarisch (Körmendy-Ékes, Lelley, Szentiványi) und - wie bereits genannt - zwei auf Deutsch (Jabloniczky, Tobler) ab, 1925 von vier zwei auf Ungarisch (Gregorovits, Szüllö) und je einer auf Deutsch (Jabloniczky) und Slowakisch (Fedor), 1929 von fünf je zwei auf Slowakisch (Dobránsky, Fedor) und Ungarisch (Hokky, Szüllö) und einer auf Deutsch (Jabloniczky), 1935 schließlich von vier drei auf Ungarisch (Esterházy, Porubszky, Szüllő) und einer auf Slowakisch (Petrášek). Da die Stimmen der OKSZP zum weitaus größten Teil auf die ungarische Bevölkerung zurückgegangen sein dürften, entsprach der Anteil der ungarischsprachigen Abgeordneten nicht der offiziellen Zahl von $5,4 \%$ (1921) und 4,8 \% (1930) Bürgern ungarischer Nationalität. ${ }^{25}$

Auf den ersten Blick instabil erscheint die Entwicklung im Bereich der Abgeordneten russischer und ukrainischer Sprache. Hier schwankte die Anzahl von eins bis sechs (0,3-2\%) bei den russischsprachigen und zwei bis fünf $(0,7-1,7 \%)$ bei den ukrainischsprachigen Abgeordneten. Neben der hier noch viel stärker zu beobachtenden Teilnahme auf Listen der großen Parteien, namentlich der Republikanischen Bauern- und Kleinlandwirtepartei (Republikánská strana zemědělského a malorolnického lidu, RSZML) und der KSČ, kam als Spezifikum die wechselseitige Austauschbarkeit der beiden Sprachen hinzu: So sprach Ivan Kurtjak 1924-1925 und 1925-1929 Ukrainisch, ehe er 1929 zum Russischen wechselte. Nikolaj Sedorjak (KSČ) sprach umgekehrt 1924-1925 Russisch, ehe er für die folgenden beiden Legislaturperioden zum Ukrainischen wechselte.

Wichtig ist deshalb zu berücksichtigen, dass im Karpatenland ganz überwiegend russinische Dialekte gesprochen wurden, von denen ein Gutteil der Linguistik das Ukrainische als Hochsprache ansah und weiter ansieht. Ohne zu dieser Frage Stellung zu beziehen, zeigen die Fälle Kurtjak und Sedorjak zumindest, dass in dieser Diskussion in der Zwischenkriegszeit kein Konsens bestand. Deshalb ergibt eine Addition der Parlamentarier russischer und ukrainischer Sprache - die Luschka im eingangs erwähnten Zitat „ruthenisch“ nannte - die etwas

25 Nationalitätenangaben zitiert nach Bohmann, Menschen, 96; Lipscher, „Parteien in der ČSR“, 219. 
stabilere Größenordnung von fünf bis neun Abgeordneten (1,7-3\%), wobei die Bandbreite damit immer noch schwankend war.

In der deutschsprachigen Literatur ist oft auf Ungerechtigkeiten in der Wahlkreisgeometrie, politikwissenschaftlich gerrymandering genannt, zu Gunsten der Tschechen und Slowaken und zu Ungunsten der Minderheiten hingewiesen worden. ${ }^{26}$ Für die deutschsprachigen Gebiete der ČSR waren die Auswirkungen allerdings so gering, dass die deutschsprachigen Parlamentarier 1925-1938 im Abgeordnetenhaus mit 24-25\% - ohne die Jahre 1933-1935 - dennoch überrepräsentiert waren, wenn als Vergleichsmaßstab die amtlichen Zahlen von 23,4 \% (1921) und 22,3 \% (1930) Deutschen mit tschechoslowakischer Staatsangehörigkeit ${ }^{27}$ herangezogen werden. Damit gelang es der deutschen Politik in der ČSR, wie auch in Lettland und übrigens auch in Polen, wo die Diskriminierung der Minderheiten im Wahlrecht offen im Sejm mit der geringeren Loyalität ebendieser Minoritäten begründet worden war, ${ }^{28}$ das auf die Schwächung der Minderheiten abzielende Wahlrecht nicht nur erfolgreich zu konterkarieren, sondern ins Gegenteil zu verkehren, sofern die amtlichen Volkszählungen als weitgehend glaubwürdig betrachtet werden.

Die wenig zutreffende Behauptung der zahlenmäßigen Diskriminierung der Minderheiten versperrt auch den Blick darauf, dass die Privilegierung nicht nationaler, sondern geografischer Natur war, da tatsächlich das Land Böhmen privilegiert war. ${ }^{29}$ Dies hatte zur Folge, dass 1925-1938 die beiden großen Sprachen Tschechisch (53,3-56 \% bei 50,8 \% [1921] und 51,1\% [1930] Staatsbürgern tschechischer Nationalität) und Deutsch stets überproportional vertreten waren. Dazu kam als Ausnahme Polnisch mit 0,7-1 \% Abgeordneten bei 0,6 \% Polen bei beiden Volkszählungen. Der Anteil der slowakischsprachigen Parlamentarier entsprach dagegen mit 14,7-15,7 \% etwa dem Anteil von 14,7 \% (1921) und 15,8 \% (1930) Slowaken - wobei an dieser Stelle noch einmal auf die OKSZP-Abgeordneten hingewiesen wird, die ihren Eid auf Slowakisch abgaben, was das Slowakische stärkte.

Klar unterproportional vertreten waren das bereits genannte Ungarische, die Ruthenen mit kumuliert 1,7-3 \% russisch- und ukrainischsprachigen

\footnotetext{
26 So ausführlich Bohmann, Menschen, 163-70. Ladislav Lipscher, Verfassung und politische Verwaltung in der Tschechoslowakei 1918-1939 (München: Oldenbourg, 1979), 68-69.

27 Nationalitätenangaben übernommen von Bohmann, Menschen, 96.

${ }^{28}$ Sejm Ustawodawczy [Verfassungsgebender Sejm]: 306. Sitzung, 10. Mai 1922, Hauptkatalog der polnischen Parlamentsbibliothek, letzter Zugriff 02.05.2016, https://bs.sejm.gov.pl/exlibris /aleph/a22_1/apache_media/FBNFNNBHQ4R4IG891N34D9KDDCCNCI.pdf.

${ }_{29}$ Zdeněk Kárník, České zeměv vére První republiky (1918-1938), Bd. 2 (Praha: Libri, 2002), 495.
} 
Abgeordneten bei einer amtlichen Bevölkerung von 3,4 \% (1921) und 3,8 \% (1930), sowie die sich in nationaler Hinsicht jüdisch nennenden Bürger der ČSR, die bei beiden Volkszählungen 1,3\% ausmachten, ${ }^{30}$ aber bis 1929 aufgrund der gestreuten Siedlung ihrer Wähler gar keine entsprechenden Abgeordneten besaßen. 1929-1938 gab es zwei jüdisch-nationale Abgeordnete, von denen 1929-1935 je einer der tschechisch- und der slowakischsprachigen Abgeordnetengruppe angehörte. 1935 entschied sich je einer für das Tschechische und das Russische. ${ }^{31}$

Wie oft benutzten die Abgeordneten der fünf Minderheitensprachen die Staatssprachen bei freier Wahl? Die Register der ersten Legislaturperiode verzeichnen dies bei drei linken Politikern: Der ungarische Sozialdemokrat József Földessy hielt seine erste Rede am 19. Juni 1920 auf Tschechisch, seine zweite am 11. November 1920 auf Deutsch, anschließend aber stets auf Ungarisch. Karl Kreibich (KSČ) gebrauchte in vier seiner 16 Wortmeldungen anstelle des Deutschen das Tschechische, erstmalig am 25. Oktober 1922. Der 1924 im Wahlkreis XXII nachgewählte Kommunist József Gáti nutzte in drei seiner vier Reden anstelle des Ungarischen das Tschechische. ${ }^{32}$ Insgesamt stehen diesen neun $(0,8 \%)$ Wortmeldungen $1.098(99,2 \%)$ gegenüber, in denen genau die Sprache gewählt wurde, in denen auch der Amtseid abgelegt worden war. Das Phänomen des Wechsels zu den Staatssprachen war also 1920-1925 eine seltene Ausnahme.

Für die Jahre 1925-1938 liegen die Daten nicht im Index aufbereitet vor. Sie müssten durch eine vollständige Durchsicht aller Stenogramme ermittelt werden. Gáti, der 1925-1929 28-mal vor dem Abgeordnetenhaus sprach, nutzte in 17 Fällen das Tschechische, wodurch er unter den zehn Parlamentariern des gesamten Abgeordnetenhauses war, die am meisten auf Tschechisch sprachen. Damit trug Gáti zur weiteren Schwächung der ohnehin verhältnismäßig schwach vertretenen ungarischen Sprache im Abgeordnetenhaus der ČSR bei. Kreibich verwandte in seinen 14 Wortbeiträgen 1925-1929 dagegen stets das Deutsche.

30 Alle Nationalitätenangaben zitiert nach Bohmann, Menschen, 96.

${ }^{31}$ III. Abgeordnetenhaus: 1. Sitzung, 12. Dezember 1929; 2. Sitzung, 13. Dezember 1929; 142. Sitzung, 14. Oktober 1931; IV. Abgeordnetenhaus: 1. Sitzung, 18. Juni 1935.

32 I. Abgeordnetenhaus: 10. Sitzung, 19. Juni 1920; 20. Sitzung, 11. November 1920; 160. Sitzung, 25. Oktober 1922; 288. Sitzung, 18. September 1924; 291. Sitzung, 24. September 1924; 303. Sitzung, 21. November 1924; 319. Sitzung, 19. Dezember 1924; 331. Sitzung, 18. März 1925; 375. Sitzung, 13. Oktober 1925. Bei den Reden Gátis entsteht dadurch im Protokoll die paradoxe Situation, dass seine Reden auf Tschechisch in ebendieser Sprache aufgeführt sind, seine auf Ungarisch gehaltenen Reden dagegen auf Slowakisch. 
Auch ohne eine exakte Bestimmung für 1925-1938 darf als sicher gelten, dass Gáti eine seltene Ausnahme war.

Werden die absoluten Anzahlen an Wortbeiträgen bei freier Sprachenwahl aller Parlamentarier nach der Abgabe des Eids - unabhängig von der tatsächlich benutzten Sprache - gruppiert (Tabellen 1-2), so zeigt dies zunächst, dass sich die Abgeordneten tschechischer und slowakischer Sprache unterdurchschnittlich ins Abgeordnetenhaus einbrachten. Bemerkenswert ist die besondere Passivität der slowakischsprachigen Parlamentarier, die erst in der letzten Legislaturperiode an Aktivitäten zulegten, an ihren Kollegen tschechischer Sprache relativ vorbeizogen und den Durchschnitt von 2,0 erreichten. Bei den kleineren Sprachgruppen hing das Engagement an Einzelpersonen, weshalb sich teilweise überaus hohe und niedrige Werte finden.

Tabelle 1: Wortmeldungen bei freier Sprachwahl, gruppiert nach Ableistung des Eides

\begin{tabular}{|l|r|r|r|r|}
\hline & $1920-1925$ & $1925-1929$ & $1929-1935$ & $1935-1938$ \\
\hline Tschechischsprachige & $1.206(46,7 \%)$ & $791(47,8 \%)$ & $1.138(51,1 \%)$ & $415(45,1 \%)$ \\
\hline Deutschsprachige & $1.010(38,9 \%)$ & $573(34,6 \%)$ & $678(30,4 \%)$ & $233(25,3 \%)$ \\
\hline Slowakischsprachige & $282(10,9 \%)$ & $165(10,0 \%)$ & $253(11,4 \%)$ & $151(16,4 \%)$ \\
\hline Ungarischsprachige & $82(3,2 \%)$ & $81(4,9 \%)$ & $86(3,9 \%)$ & $67(7,3 \%)$ \\
\hline Ukrainischsprachige & $9(0,3 \%)$ & $31(1,9 \%)$ & $6(0,3 \%)$ & $13(1,4 \%)$ \\
\hline Russischsprachige & $6(0,2 \%)$ & $3(0,2 \%)$ & $32(1,4 \%)$ & $24(2,6 \%)$ \\
\hline Polnischsprachige & - & $12(0,7 \%)$ & $37(1,6 \%)$ & $17(1,8 \%)$ \\
\hline Zusammen & $\mathbf{2 . 5 9 5}$ & $\mathbf{1 . 6 5 6}$ & $\mathbf{2 . 2 2 7}$ & $\mathbf{9 2 0}$ \\
\hline
\end{tabular}

Quelle: eigene Zählung.

Überraschungen bietet auch das Verhalten der deutschsprachigen Abgeordneten: In den 1920er Jahren war ihr Engagement vor dem Plenum enorm hoch, in den Jahren 1920-1925 waren sie sogar quantitativ von der Parität mit dem Tschechischen nicht weit entfernt. In den 1930er Jahren gingen die Wortmeldungen deutschsprachiger Abgeordneter dagegen deutlich und fast bis auf den Durchschnittswert zurück, was sich sowohl quantitativ als auch qualitativ niederschlug. Diese Entwicklung betraf die gesamte Gruppe deutschsprachiger Abgeordneter und ist nicht etwa nur dem großen Wahlerfolg der negativistischen SdP 1935 geschuldet. Die durchschnittliche Anzahl an Wortmeldungen lagen vielmehr bei den Parteien SdP, DSAP, DCSVP und dem Bund der Landwirte (BdL) nahezu gleichauf. Lediglich der Wert der fünf deutschsprachigen Kommunisten weicht stark nach oben ab. 
Tabelle 2: Wortmeldungen bei freier Sprachwahl pro Mandat und 100 Sitzungen

\begin{tabular}{|l|c|c|c|c|}
\hline & $1920-1925$ & $1925-1929$ & $1929-1935$ & $1935-1938$ \\
\hline Tschechischsprachige & 2,0 & 2,3 & 1,8 & 1,6 \\
\hline Deutschsprachige & 3,5 & 3,6 & 2,5 & 2,1 \\
\hline Slowakischsprachige & 1,8 & 1,7 & 1,5 & 2,0 \\
\hline Ungarischsprachige & 2,5 & 3,7 & 3,3 & 4,4 \\
\hline Ukrainischsprachige & 3,7 & 2,9 & 0,7 & 3,0 \\
\hline Russischsprachige & 1,6 & 1,4 & 2,9 & 2,8 \\
\hline Polnischsprachige & - & 2,8 & 3,3 & 5,7 \\
\hline Durchschnitt & $\mathbf{2 , 4}$ & $\mathbf{2 , 6}$ & $\mathbf{2 , 0}$ & $\mathbf{2 , 0}$ \\
\hline
\end{tabular}

Quelle: eigene Zählung.

Erläuterung: Da die vier Legislaturperioden unterschiedlich lang waren, können die Daten aus Tabelle 1 nicht miteinander verglichen werden. Um Vergleichbarkeit zu ermöglichen, wird hier ein Zeitraum von 100 Sitzungen genommen. Die angegebenen Werte zeigen an, wie oft sich ein einzelner Abgeordneter aus allen Sprachgruppen in 100 Sitzungen im Schnitt zu Wort meldete.

Neben Wortmeldungen vor dem Plenum, bei denen die Sprache frei gewählt werden konnte, gab es solche, bei denen zwingend das Tschechoslowakische zu gebrauchen war. Die mit Abstand umfangreichste dieser Gruppe an Wortmeldungen war das Halten eines Gesetzesreferates für ein Gesetzesvorhaben. 1920-1925 entfielen 1.231 Gesetzesreferate ${ }^{33}$ auf tschechisch- $(90,4 \%)$ und 130 auf slowakischsprachige Abgeordnete (9,6 \%). 1925-1929 lag die Verteilung bei 715 Referaten tschechisch- $(83,7 \%)$ und 139 slowakischsprachiger Parlamentarier (16,3 \%). 1929-1935 lag sie bei 1.022 Referaten von Deputierten tschechischer (88,3\%) und 135 slowakischer Sprache (11,7 \%). 1935-1938 entfielen 354 Gesetzesreferate auf tschechisch- (84,1\%), 60 auf slowakisch$(14,3 \%)$, fünf auf russisch- $(1,1 \%)$ und je eins auf deutsch- und ukrainischsprachige Abgeordnete (je 0,2\%).

Die Betrachtung der Gesetzesreferate erklärt, warum sich die Gruppen der tschechisch- und slowakischsprachigen Abgeordneten so wenig im Bereich der Wortmeldungen mit freier Sprachenwahl einbrachten: Sie waren in erheblichem Ausmaß mit der Gesetzgebung beschäftigt. Dabei war die Beteiligung der tschechischsprachigen Abgeordneten noch einmal signifikant höher als die der slowakischsprachigen. Anders als in Lettland, wo sich 1923-1934 die Parlamentarier deutscher, jüdischer und polnischer Nationalität auch sehr stark

33 Die in diesem Bereich genannten Ziffern beziehen sich ausschließlich auf die Gesetzesreferate, nicht auf die damit tatsächlich verbundenen Wortmeldungen. 
als Gesetzesreferenten in lettischer Sprache einbrachten, ${ }^{34}$ begann die Zeit der Gesetzesreferate durch Minderheitenabgeordnete in der ČSR erst am 21. Januar 1937 mit einem gemeinsamen Referat der beiden Abgeordneten der Jüdischen Partei (Židovská strana, ZS), dem tschechischsprachigen Angelo Goldstein und dem ansonsten russischsprachigen Chaim Kugel. Goldstein und Kugel brachten dabei gemeinsam einen Gesetzentwurf über die jüdischen Religionsgemeinschaften in den Ländern Böhmen und Mähren-Schlesien ein. ${ }^{35}$

Das Beispiel Kugels machte Schule: 1937-1938 wurden in gewissem Umfang Gesetzesreferate von Abgeordneten der Minderheiten in tschechischer Sprache üblich, die den Koalitionsparteien angehörten. Vor allem der ansonsten russischsprachige Pavel Kossey (RSZML) trat mit drei Gesetzesreferaten in Erscheinung. Je ein Gesetzesreferat hielten Irene Kirpal (DSAP) und der ansonsten ukrainischsprachige Julian Révay von der Tschechoslowakischen Sozialdemokratischen Arbeiterpartei (Československá sociálně demokratická strana dělnická, ČSDSD). Die Übernahme von Gesetzesreferaten durch Vertreter der Minderheiten hätten bei einem Weiterbestehen der ČSR einen Schritt zur verstärkten Partizipation darstellen können. Zumindest sind sie ein augenfälliger Beweis dafür, dass die Bereitschaft zum Aktivismus nach wie vor bei manchen vorhanden war.

Ein bemerkenswertes Novum stellte das Gesetzesreferat Kosseys (RSZML) vom 19. November 1938 über die Autonomie des Karpatenlandes dar. Dabei handelte es sich zum einen um das einzige Gesetzesreferat eines Politikers der Minderheiten in der kurzen Zweiten Tschechoslowakischen Republik. Entgegen der bisherigen Praxis erfolgte es zudem auf Russisch, ${ }^{36}$ ein Vorgang von nicht zu unterschätzender Symbolik: Waren zuvor im Karpatenland wie in anderen Regionen auch die Minderheitensprachen Amtssprache, wenn sie einen Anteil von mindestens $20 \%$ unter der lokalen Bevölkerung hatten, so wertete Kossey das Russische nun im Zuge des Autonomiegesetzes zur landesweiten zusätzlichen Staatssprache auf.

Schlussendlich werden nun alle Wortmeldungsformen unbeachtet der Tatsache, ob sie nur in der Staatssprache oder in allen Sprachen erfolgen durften,

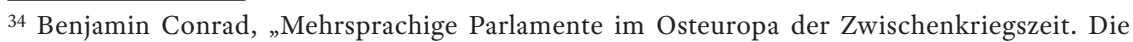
Beispiele Lettland und Tschechoslowakei. Bestandsaufnahme und Vergleich“, in Parlamentarier der deutschen Minderheiten im Europa der Zwischenkriegszeit, hrsg. v. Benjamin Conrad, Hans-Christian Maner und Jan Kusber (Düsseldorf: Droste, 2015), 117-36, hier 133. Conrad, Loyalitäten, 52, 173.

35 IV. Abgeordnetenhaus: 76. Sitzung, 21. Januar 1937.

36 IV. Abgeordnetenhaus: 155. Sitzung, 19. November 1938. 
zusammengerechnet. Dabei ergibt sich, dass die tschechischsprachige Parlamentariergruppe insgesamt diejenige mit der höchsten durchschnittlichen Zahl an Wortmeldungen war. Durch die Nichtwahrnehmung von Gesetzesreferaten durch die Abgeordneten der Minderheitensprachen bis 1937 kann dies auch nicht überraschen. Auch in Lettland 1923-1934 waren die Deputierten deutscher, jüdischer und polnischer Nationalität durch die Übernahme von Gesetzesreferaten in das parlamentarische System signifikant besser eingebunden als diejenigen russischer Nationalität, die sich den Gesetzesreferaten mit nur einer Ausnahme verweigerten.

\section{Schluss: Mehrsprachigkeit des Parlaments als Faktor der Partizipation?}

Der vergleichende Blick auf das mehrsprachige Abgeordnetenhaus der Tschechoslowakei zeigt, dass die Frage nach der Mehrsprachigkeit als Faktor der Partizipation kein einheitliches Bild abgibt. Sicher ist, dass dem Abgeordnetenhaus die Mehrsprachigkeit nicht geschadet hat. Schließlich war die ČSR derjenige Staat in Ostmittel- und Südosteuropa, in dem die parlamentarisch-demokratische Ordnung bis zum Zusammenbruch 1938 am längsten währte. Sie bewies damit auch - ganz entgegen den vielfach geäußerten zeitgenössischen Befürchtungen und Behauptungen -, dass zahlen- und anteilsmäßig große Minderheiten nicht ausschlaggebend für den Bestand des parlamentarischen Systems waren.

Wichtigste Erkenntnis aus der Betrachtung des Datensatzes ist, dass die Minderheiten sich trotz der international vergleichsweise liberalen Sprachregelung geringer im Abgeordnetenhaus einbrachten als die Abgeordneten der tschechischen Sprachgruppe. Bei den kleineren Sprachen kam es immerhin ganz überwiegend zu überdurchschnittlichen Wortmeldungen in der Muttersprache, aber zu nahezu keiner weiteren Partizipation.

Bei den deutschsprachigen Abgeordneten kam es zu einem auffälligen Rückgang an Wortmeldungen. Wohl hatte der Einfluss der „Burg“ (Hrad), also des Lagers um die Präsidenten Tomáš Garrigue Masaryk (1918-1935) und Edvard Beneš (1935-1938), oder das Weiterwirken des pětka-Gedankens, gemeint ist der in der ersten Hälfte der 1920er Jahre tätige Ausschuss der fünf wichtigsten tschechoslowakischen Parteien, das Interesse am Abgeordnetenhaus sinken lassen. Zudem ließen sich auch durch den Regierungseintritt der DCSVP und des BdL 1926 und später der DSAP keine substantiellen Fortschritte in Sachen Rechte der deutschsprachigen Bevölkerung erreichen, weshalb parlamentarisch 
seit den Wahlen von 1935 unter den deutschsprachigen Abgeordneten der Negativismus, repräsentiert durch die SdP, überwog. ${ }^{37}$

Doch dürfen die Ursachen nicht allein auf der Seite des tschechoslowakischen Staates gesucht werden. In der ČSR beherrschten 1920 vielleicht ein Sechstel der deutschen Abgeordneten das Tschechische. ${ }^{38}$ In Lettland beherrschten Ende 1918 ganz ähnlich nur zwei der ersten acht deutschen Abgeordneten Lettisch fließend. ${ }^{39}$ In der Legislaturperiode 1922-1925 beherrschten jedoch, nach intensivem Lernen, bereits alle deutschen Parlamentarier Lettlands das Lettische fließend. Dadurch konnten sie alle Positionen im Staat einnehmen, weshalb gute Kenntnisse des Lettischen fortan für alle deutschen Kandidaten zu einer Voraussetzung wurden. ${ }^{40}$

In der ČSR gab es keine vergleichbare Entwicklung des Spracherwerbs unter den deutschsprachigen Abgeordneten: 1927 gaben von den 73 Abgeordneten und Senatoren des BdL, der DCSVP, der Deutschen Nationalpartei, der DNSAP und der Deutschen Gewerbepartei $51 \%$ an, dass ihnen die tschechische Sprache gänzlich unbekannt sei. Weitere $26 \%$ gaben an, die Sprache radebrechend zu beherrschen, $15 \%$ gaben fließende und $8 \%$ vollständige Beherrschung an. ${ }^{41}$ Wären die deutschen Sozialdemokraten und Kommunisten 1927

37 Manfred Alexander, „Die ,Burg“ und die Deutschen“, in Die „Burg“. Einflußreiche politische Kräfte um Masaryk und Beneš, Bd. 2, hrsg. v. Karl Bosl (München: Oldenbourg, 1974), 59-78, hier 74-78; Alexander, „Leistungen“, 74, 77, 84-87; Freia Anders, „Verfassungswirklichkeit und Verfassungskritik in der Ersten Tschechoslowakischen Republik“, in Demokratie in der Krise. Europa in der Zwischenkriegszeit, hrsg. v. Christoph Gusy (Baden-Baden: Nomos, 2008), 229-62; Eva Broklová, „Die Interpretation der Probleme des politischen Systems der Ersten Tschechoslowakischen Republik durch drei deutsche Historiker“, in Bohemia 39 (1998): 382-96, hier 393; Hoensch, Tschechoslowakei, 53-60; Kárník, České země, Bd. 1, 139-52, 407-16; Kracik, Aktivismus, 434-44; Heinrich Kuhn, „Der Anteil der Deutschen an der ,Burg““, in Die „Burg“. Einflußreiche politische Kräfte um Masaryk und Beneš, Bd. 1, hrsg. v. Karl Bosl (München: Oldenbourg, 1973), 109-28, hier 124-28; Daniel E. Miller, Forging Political Compromise. Antonín Švehla and the Czechoslovak Republican Party 1918-1933 (Pittsburgh: University of Pittsburgh Press, 1999), 70-78; Slapnicka, „Länder“, 34-35; Andreas Wirsching, „Verfassung und Verfassungskultur der Zwischenkriegszeit“, in Demokratie in der Krise. Europa in der Zwischenkriegszeit, hrsg. v. Christoph Gusy (Baden-Baden: Nomos 2008), 371-89, hier 378-79.

38 Kučera, Minderheit, 214.

39 Dies widerlegt auch die Annahme von Balling, der den Deutschbalten unterstellt, aufgrund ihrer Herkunft aus einer regionalen Minderheit, Kenntnisse des Lettischen gehabt zu haben. Vgl. Mads Ole Balling, „Zur soziokulturellen Struktur der deutschen Abgeordneten in der Tschechoslowakei und anderen ostmitteleuropäischen Staaten 1919-1945“, Bohemia 36 (1995): 39-56, hier 43.

40 Conrad, Loyalitäten, 52.

${ }^{41}$ Lipscher, Verfassung, 66. Bei dieser Befragung wurden Senatoren und Abgeordnete gemeinsam befragt, eine nachträgliche Trennung beider Gruppen ist nicht möglich. Es wird davon ausgegangen, dass es keine signifikanten Unterschiede zwischen deutschsprachigen Senatoren und Abgeordneten in Hinblick auf die Sprachkenntnis gegeben hat. 
mit einbezogen worden, wäre das Verhältnis dank der Kenntnisse von Politikern wie Ludwig Czech (DSAP) und Karl Kreibich (KSČ) wahrscheinlich etwas besser ausgefallen, zumal die DSAP für ihre Abgeordneten gleich 1920-1921 Tschechisch-Sprachkurse organisiert hatte. Insgesamt waren die Kenntnisse des Tschechischen über die 1920er Jahre hinweg unter den deutschen Parlamentariern dennoch nur geringfügig ansteigend. ${ }^{42}$

Selbstverständlich ist die Zahl der fünf bis sechs deutschen Parlamentarier Lettlands, wo es oft auf jeden ankam, nicht mit der Zahl von 66-76 deutschen Abgeordneten in der ČSR vergleichbar. Das Beispiel Lettland zeigt jedoch, dass eine voll integrationsbereite Parlamentariergruppe auch im linguistischen Bereich anders agierte. Dies stellt die zweite Erkenntnis dar: Durch die Weigerung vieler deutscher Abgeordneter, das Tschechische zu erlernen, leisteten diese ihrerseits einen Beitrag zum Fortbestehen der Partizipationshürden. Selbst bei voller Gleichberechtigung der drei großen Sprachen Tschechisch, Deutsch und Slowakisch im Abgeordnetenhaus hätten die deutschsprachigen Abgeordneten im parlamentarischen Alltag weiter mit Verständnisproblemen zu kämpfen gehabt. Die Übernahme von Gesetzesreferaten, Regierungsämtern oder von Posten im Parlamentsvorstand hätte sich für solche Abgeordnete weiter schwierig gestaltet.

Warum verweigerten sich viele deutsche Abgeordnete in der ČSR dem Spracherwerb? Abgesehen von individuellen Gründen wie fortgeschrittenem Alter ist bei Angehörigen einer überwiegend höher gebildeten, ehemaligen Führungsschicht von einer eher negativen Hypothese auszugehen, deren Erforschung ein Desiderat darstellt. In den eingangs zitierten Erinnerungen Luschkas gibt es dazu Hinweise: Gedanken an die Anerkennung des Statusverlusts, generelle Unzufriedenheit mit der Pariser Ordnung und daraus resultierende Vorbehalte gegen die ČSR oder schlicht kulturelle Überlegenheitsgefühle gegenüber den Tschechen bis hin zu Revisionismus bei der SdP mögen die Gründe gewesen sein. Deshalb war es symptomatisch, aber auch nicht überraschend, dass der SdP-Fraktionsvorsitzende Karl Hermann Frank kein Tschechisch verstand. ${ }^{43}$

Sollte sich die genannte Hypothese als zumindest in großen Teilen stichhaltig herausstellen, so wäre für die beschränkte Partizipation deutscher Abgeordneter im Abgeordnetenhaus der Tschechoslowakei ein Wechselspiel zwischen im internationalen Vergleich weitgehenden, aber dennoch von deutscher Seite als ungenügend empfundenen Sprachenrechten, in Kombination mit Defiziten

42 Kučera, Minderheit, 214.

43 Balling, „Struktur“, 44. 
in der Verfassungswirklichkeit einerseits mit allen Arten von eigenen Vorbehalten der deutschen Seite gegen die neue politische Ordnung andererseits verantwortlich gewesen. 